\title{
Effect of Overlapping Intrinsic Spin Resonances on e-Lens Lattices from FY13 Polarized Proton Run
}

\author{
Vahid H. Ranjbar \\ Collider Accelerator Department, Brookhaven National Lab, \\ Upton, NY 117r7, United States of America \\ vranjbar@bnl.gov
}

Published 29 February 2016

\begin{abstract}
During the 2013 Polarized Proton run RHIC was run with a lattice designed to accommodate the phases necessary for the Electron lens. This lattice resulted in significant changes in magnitude and distribution of the intrinsic resonances. The overall magnitude of the strong intrinsic resonances were smaller, however the neighbouring weak intrinsic resonances grew. We present results from $2 \mathrm{D}$ direct tracking with 5,000 particles and 2D lattice independent tracking .
\end{abstract}

\section{Indroduction}

One of the major limitations for high intensity colliders are due to the head on beam-beam collisions which with a high enough intensity can lead to emittance blow-up. In order to compensate this effect an electron lens ${ }^{1}$ has been installed in the Relativistic Heavy Ion Collider (RHIC). In order for this compensation to work effectively however, the betatron phase advance between the collisional interaction point (IP) and the e-lens should be fixed at 90 degrees. To accommodate this phase advance new lattices (e-lens lattices) were developed for both the Blue and Yellow ring.

During the $2013255 \mathrm{GeV}$ polarized proton run the new e-lens lattices for both rings were commissioned. The e-lens lattice required different integer tunes for both rings. For the Blue ring the integer tune went from $\left(Q_{x}=28, Q_{y}=29\right)$ to $\left(Q_{x}=\right.$ $\left.27, Q_{y}=29\right)$ and for the Yellow ring, from $\left(Q_{x}=28, Q_{y}=29\right)$ to $\left(Q_{x}=29, Q_{y}=\right.$ 30). Compared with the run 2012 lattice (standard lattice), both the yellow and blue ring e-lens lattices had lower values for the three very strong spin intrinsic resonances above $100 \mathrm{GeV}$ (see Table. 1). However the two neighboring weak resonances were larger than the run 2012 lattice. This was due in part to the effect of the broken symmetry imposed by the e-lens lattice (see Fig. 1).

This is an Open Access article published by World Scientific Publishing Company. It is distributed under the terms of the Creative Commons Attribution 3.0 (CC-BY) License. Further distribution of this work is permitted, provided the original work is properly cited. 
Table 1. Difference of e-lens lattice and standard lattice three strongest intrinsic resonances.

\begin{tabular}{l|c|r} 
Resonances in $\mathrm{G} \gamma$ & Blue (new-old) & Yellow (new-old) \\
\hline $231+\nu_{y}$ & -0.0387 & -0.0415 \\
$411-\nu_{y}$ & -0.06134 & -0.0655 \\
$393+\nu_{y}$ & -0.05347 & -0.0347
\end{tabular}
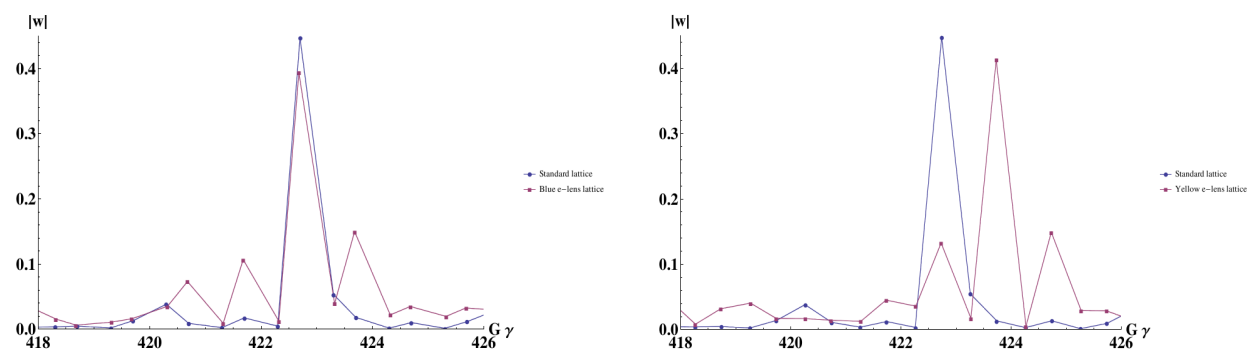

Fig. 1. Comparison of e-lens intrinsic resonance strengths with standard RHIC lattice for $393+\nu_{y}$

This was the first time the RHIC lattice had run with significantly altered intrinsic spin resonance structure. Initially it was expected that the polarization transmission through the RHIC ramp would improve since, individually all the intrinsic resonances were well below the depolarization threshold in the presence of two snakes. This threshold was understood by previous theoretical studies, ${ }^{2},{ }^{3}$ however these studies didn't account for how changes in the overlap between nearby intrinsic resonances might effect the final polarization aperture.

The results from the 2013 polarized proton run, in fact didn't show strong evidence of improvement as was expected and in fact polarization transmission on the ramp may have suffered. To understand this effect we employed the newly developed teaspink ${ }^{4}$ code to perform 2D tracking $\left(\epsilon_{s}=\epsilon_{x}=0\right)$ on an mpi-gpu platform. Additionally a python code was developed to perform 2D Unitary integration over multiple resonances in a lattice independent manner. These studies motivated us to re-visit the theory and carefully analyze the drivers of depolarization during an intrinsic resonance crossing with single and multiple resonances. While there has been much effort in past years devoted to understanding polarization behavior in the presence of snakes there were several questions which still remained either unanswered or unclear from the current literature.

For example, from our reading of the literature it seemed to be unclear as too whether in an error free lattice with two orthogonal snakes and a single resonance (the so called single resonance model (SRM)) there was any mechanism for polarization loss when the betatron tunes are irrational. There was much discussion of so called critical resonance thresholds being either at 1 or 0.5 depending on the author. $^{2,5}$ Yet we were to understand that the fact that our spin tune remained amplitude independent ${ }^{5}$ for this system meant there was no mechanism for polarization loss. Indeed other authors pointed to discontinuities in the spin tune as 
signatures for polarization loss. ${ }^{6}$ So after considering the single resonance model under resonances strengths $(0$ - 5) over a whole range of irrational tunes (0-1.0) our conjecture is that provided we begin tracking on the stable spin direction and compare final spin orientation with the final stable spin direction that there is no mechanism to move the spin vector away from the stable spin direction for acceleration rates common in RHIC . This conjecture is based on our tracking with the single resonance model with orthogonal snakes and the understanding that for this system the spin tune is amplitude independent. A weaker statement is that, at least for the orthogonal system of snakes with a single resonance at an irrational betatron tune, there is no polarization loss observable for any resonance strength which could fit into the RHIC aperture.

A secondary question follows from the first, which is if the two orthogonal snake SRM model has an effectively infinite polarization aperture for irrational tunes, how does this aperture respond as secondary resonances are introduced. What are the thresholds in strength and distance from the main resonance? What role does phase play?

\section{Bench Marking Lattice Independent Tracking}

We have developed a python code to integrate the $2 \mathrm{D}$ spinor form of the ThomasBMT equation:

$$
\frac{d \Psi}{d \theta}=-\frac{i}{2}\left(\begin{array}{c}
f_{3}-\xi \\
\xi^{*}-f_{3}
\end{array}\right) \Psi .
$$

Where $\xi(\theta)=F_{1}-i F_{2}$ and $f_{3}=\left(1+F_{3}\right)$ with,

$$
\begin{aligned}
& F_{1}=-\rho z^{\prime \prime}(1+G \gamma) \\
& F_{2}=(1+G \gamma) z^{\prime}-\rho(1+G)\left(\frac{z}{\rho}\right)^{\prime} \\
& F_{3}=-(1+G \gamma)+(1+G \gamma) \rho x^{\prime \prime} .
\end{aligned}
$$

This follows from a standard derivation in. ${ }^{2}$

Using a 4th order Magnus Gaussian quadrature integrator described in ${ }^{7}$ our code can integrate Eq. 1 for an arbitrary $\xi(\theta)$. Where the snakes are added into the lattice as thin spin kicks.

We first consider the polarization response for a single isolated resonance. This has been much studied in the past especially the non-accelerating case where a analytical expression and approximations have been developed by S. Mane. ${ }^{5}$ We re-visit this as both a cross check of our new integrator (see Fig. (2)). Next we compare the accelerating case to see if we can reproduce similar results as the direct tracking while crossing the $393+\nu_{y}$ resonance for the standard lattice, Blue e-lens e-lens.

As we can see in Figs. 3, the performance of the simple 2D lattice independent tracker seemed to perform reasonably using four resonances between $\pm 1 G \gamma$ from 


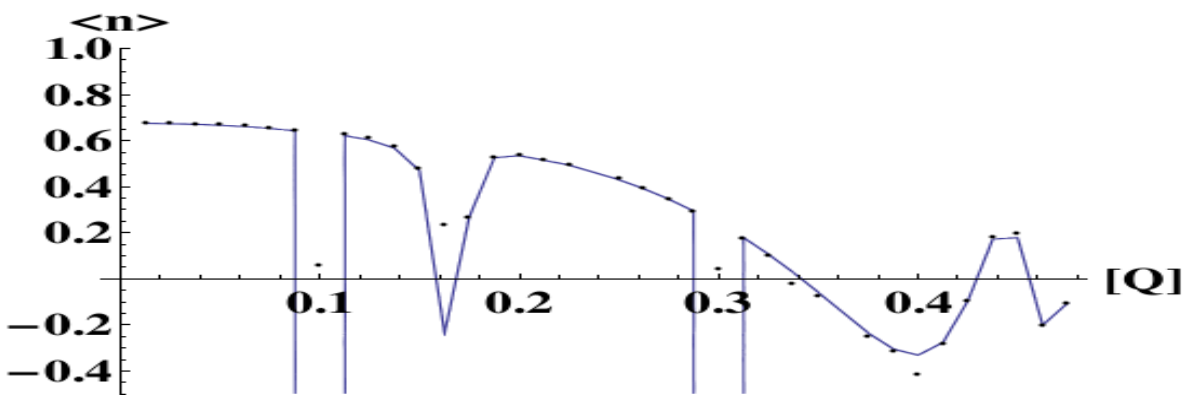

Fig. 2. Plot of stroboscopic average vertical component $\langle n\rangle$ versus the fractional orbital tune $[Q]$ with resonance amplitude of 0.4 . Dots represent numerically calculated using 8 particle average and lines are the analytically calculated values using S. Mane's formula.
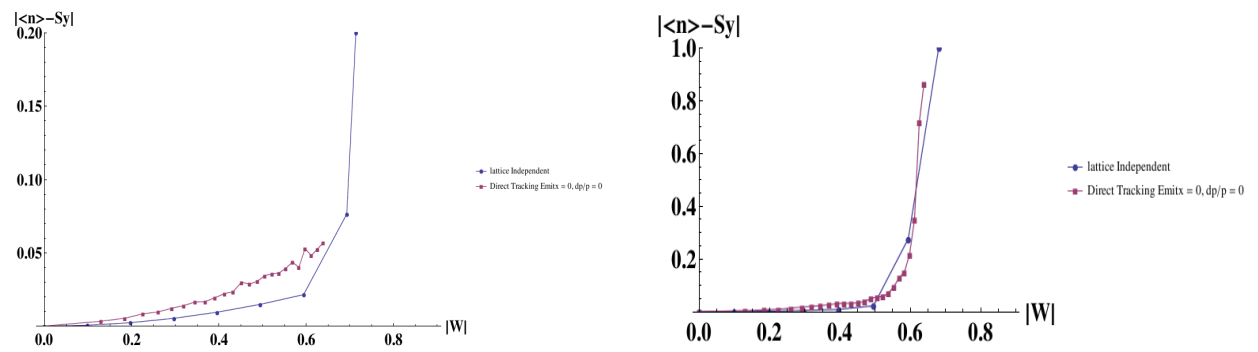

Fig. 3. Comparing 2D lattice independent tracking to direct tracking for the Standard lattice crossing the $393+\nu_{y}$ resonances. Here the lattice independent code used 5 resonances between $\pm 1 G \gamma$ from the central resonance. For the direct tracking we set the horizontal and longitudinal emittance to zero. The acceleration rate is $\frac{d \gamma}{d t}=1.15 / \mathrm{sec}$. We re-scaled the vertical emittance to absolute resonance strength $|\mathrm{W}|$, based on the strength of the main resonance at $10 \pi \mathrm{mm}-\mathrm{mrad}$ as calculated by DEPOL.

the central resonance. We attribute the differences due to two possible sources; the initial or final stroboscopic averages were not completely converged or vertical precession due to the quadrupoles since this model neglects the $(1+G \gamma) \rho x^{\prime \prime}$ term in the Thomas-BMT (Bargmann, Michel, and Telegdi) equation.

In Fig. 4 we can see a qualitatively similar response for the different lattices compared to the direct tracking.

\section{Effect of Overlapping Resonances}

The introduction of a second resonance dramatically changes the depolarization threshold and curve structure. The nature of this response depends on relative distance and phase between the primary and secondary resonance. The phase response can be seen in Fig. 5 where we can see polarization response to relative phase between the strong and weak neighboring intrinsic spin resonance when $\Delta K=-1,1,0.346$ representing the three neighbooring intrinsic resonances. The 

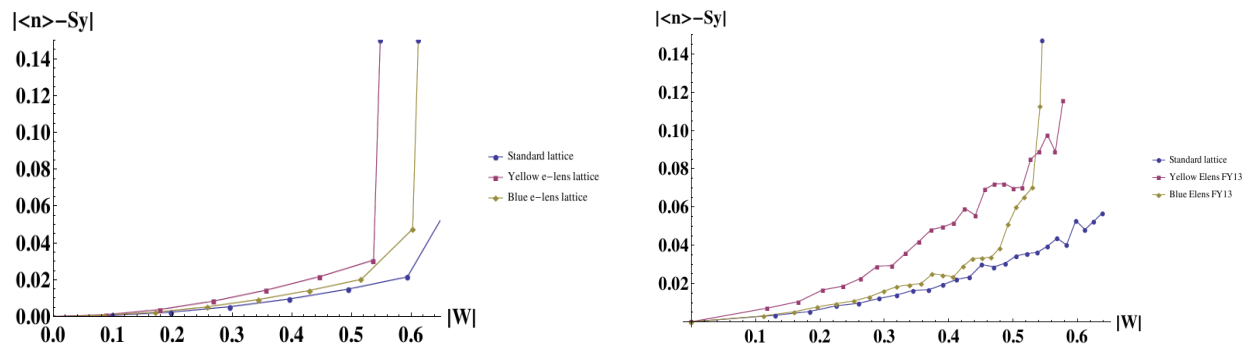

Fig. 4. (top) 2D lattice independent for the Standard, Yellow and Blue e-lens lattice crossing the $393+\nu_{y}$ resonances. Here the lattice independent code used 5 resonances between $\pm 1 G \gamma$ from the central resonance. The fractional tune is $\nu_{y}=0.671$ and acceleration rate is $\frac{d \gamma}{d t}=1.15 / \mathrm{sec}$. We re-scaled the vertical emittance to absolute resonance strength $|\mathrm{W}|$, based on the strength of the main resonance at $10 \pi m m-m r a d$ as calculated by DEPOL.(bottom) Same only with direct tracking and emittances re-scaled to the strength of main resonance.

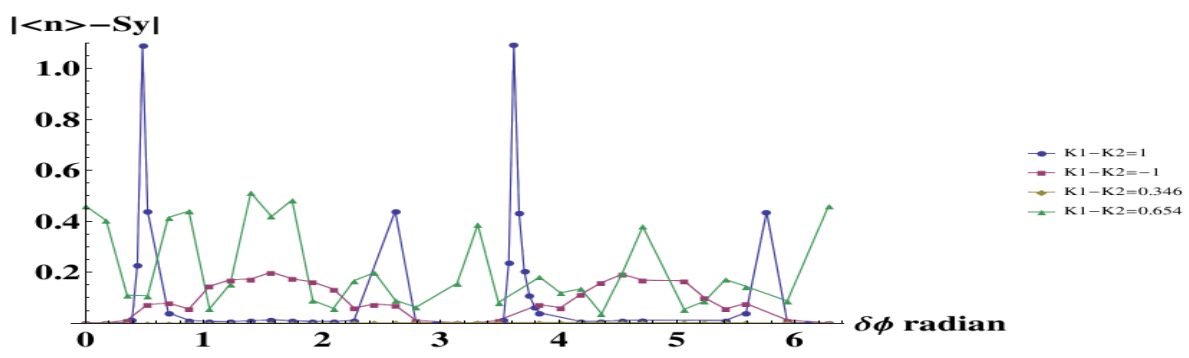

Fig. 5. Polarization response for particle at $\epsilon_{K_{1}}=0.587$ and $\epsilon_{K_{2}}=0.15$. The resonances are separated by $\Delta G \gamma=-1,1,0.346$ representing the neighboring three intrinsic resonances. The relative phase between the two resonances are scanned between 0 and $2 \pi$. Acceleration rate is nominal RHIC ramp rate $d \gamma / d t=1.12$ and fractional vertical tune $Q y=0.673$.
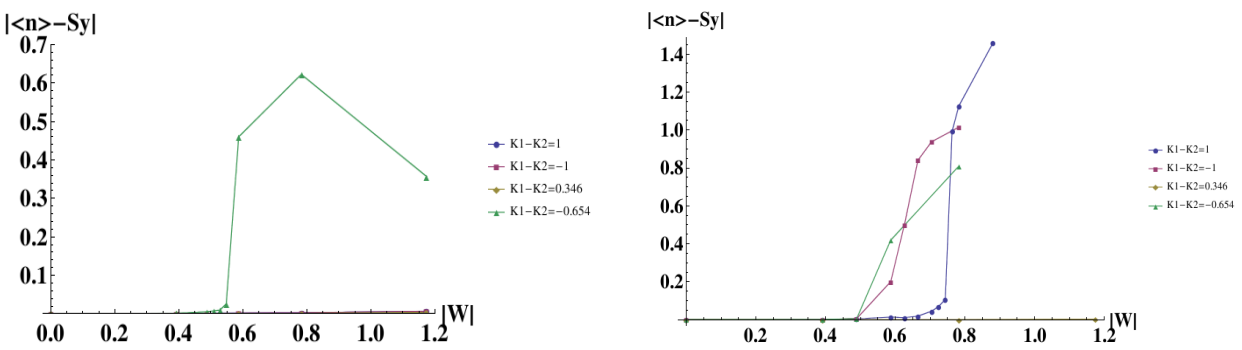

Fig. 6. Polarization response to resonance strength for 8 particles with base $\epsilon_{K_{1}}=0.3914$ and $\epsilon_{K_{2}}=0.1$. The magnitude of both resonances are then scaled together. Two resonances are considered seperated by $\Delta G \gamma=-1,1,0.654,0.346$ representing the neighboring four intrinsic resonances. The relative phase between the two resonances are 0 (top) and $\pi / 2$ (bottom). Acceleration rate is nominal RHIC ramp rate $d \gamma / d t=1.12$ and fractional vertical tune $Q y=0.673$. 
strongest effect occurs at \pm 1 unit of $G \gamma$ while the effect of the non-integer appears neglible. For these cases the effect of the phase appears symetric about $\pi$.

Next we consider the resonance amplitude response as resonances at different $\Delta G \gamma$ and different phases are introduced. In Fig. (6) we see that the $\delta \phi=\pi / 2$ have much larger effect than $\delta \phi=0$ and $K_{1}-K_{2}=-1$ has an earlier effect than $K_{1}-K_{2}=1$ (consistent with Fig. (5)). So clearly the introduction of a secondary resonance can reduce the depolarization threshold which is strongly dependent on the relative phase between the two intrinsic resonances.

\section{Acknowledgments}

Work supported the URA., Inc., under contract DE-AC02-76CH03000 with the U.S. Dept. of Energy. This research used resources of the National Energy Research Scientific Computing Center, which is supported by the Office of Science of the U.S. Department of Energy under Contract No. DE-AC02-05CH11231

\section{References}

1. X. Gu, F.Z. Altinbas, E. Beebe et. al., The electron lens test bench for the relativistic heavy ion collider at Brookhaven National Laboratory, NIM Section A: Accelerators, Spectrometers, Detectors and Associated Equipment, Volume 743, 11 April 2014, Pages 56-67

2. S. Y. Lee, Spin Dynamics and Snakes in Synchrotrons, World Scientific Pub Co Inc, 1997.

3. Design Manual - Polarized Proton Collider at RHIC, BNL (1998); http://www. agsrhichome.bnl.gov/RHIC/Spin/.

4. D. Abell and D. Messier and V. Ranjbar and D. Barber, Accurate and efficient spin integration for particle accelerators, Phys. Rev. ST-AB. (2013) to be published.

5. S. R. Mane, A critical analysis of the conventional theory of spin resonances in storage rings, NIM Section A: Accelerators, Spectrometers, Detectors and Associated Equipment, 528(3) 677-706 (2004).

6. M. Vogt, Ph.D. Thesis, University of Hamburg, also available as DESY-THESIS-2000054, 2000

7. S. Blanes and F. Casas and J. A. Oteo and J. Ros, The Magnus expansion and some of its applications, Physics Reports 470, 151-238 (2009) 